02

\title{
Снятие электродинамического запрета и особенности спектров гигантского комбинационного рассеяния в фуллерене $\mathbf{C}_{70}$
}

\author{
(C) А.М. Полуботко ${ }^{1}$, В.П. Челибанов ${ }^{2}$ \\ ${ }^{1}$ Физико-технический институт им. А.Ф. Иофрфе РАН, \\ 194021 Санкт-Петербург, Россия \\ 2 Университет ИТМО, \\ 197101 Санкт-Петербург, Россия \\ e-mail: alex.marina@mail.ioffe.ru, Chelibanov@gmail.com
}

Поступила в редакцию 27.10.2017 г.

\begin{abstract}
Показано, что в фуллерене $\mathrm{C}_{70}$, который может в каком-то смысле рассматриваться как деформированный фуллерен $\mathrm{C}_{60}$, происходит снятие электродинамического запрета сильного квадрупольного взаимодействия света с молекулами, который реализуется в фуллерене $\mathrm{C}_{60}$, в силу понижения симметрии с икосаэдрической группы $Y_{h}$ до группы симметрии $D_{5 h}$. Это приводит к появлению линий в спектре гигантского комбинационного рассеяния (ГКР), запрещенных в обычном комбинационном рассеянии (КР), а также запрещенных в спектре ГКР фуллерена $\mathrm{C}_{60}$, и активных в спектрах инфракрасного поглощения. Экспериментально измеренный спектр ГКР $\mathrm{C}_{70}$ показал присутствие таких линий, что полностью подтверждает наши представления о дипольно-квадрупольном механизме ГКР.
\end{abstract}

DOI: $10.21883 /$ OS.2018.04.45745.252-17

Исследование фуллеренов методом гигантского комбинационного рассеяния света (ГКР) представляет большой интерес, поскольку может дать новую важную информацию об их структуре и оптических свойствах. Кроме того, как оказалось $[1,2]$, в фуллерене $\mathrm{C}_{60}$ (рис. $1, a)$ хорошо прослеживается сам механизм ГКР. Это выражается в том, что в этой молекуле реализуется так называемый электродинамический запрет сильного квадрупольного взаимодействия, когда квантовомеханический оператор сильного квадрупольного взаимодействия тождественно равен нулю в силу принадлежности $\mathrm{C}_{60}$ икосаэдрической группе симметрии $Y_{h}$ и в силу закона электродинамики $\operatorname{div} \mathbf{E}=0[1,2]$. В спектрах ГКР электродинамический запрет проявляется в отсутствии линий, относящихся к колебаниям, преобразующимся по трехмерному неприводимому представлению $T_{1 u}$ (рис. 2), которое описывает трансформационные свойства компонент дипольного момента $d_{e, x}, d_{e, y}$ и $d_{e, z}$. Как известно, эти линии запрещены в обычном комбинационном рассеянии (КР) и разрешены в инфракрасном поглощении. Однако они становятся разрешенными и в ГКР. В фуллерене $\mathrm{C}_{60}$ эти линии становятся также запрещенными, но уже в силу электродинамического запрета $[1,2]$. В настоящей работе мы хотим обратить внимание на то, что в фуллерене $\mathrm{C}_{70}$, (рис. $\left.1, b\right)$, который в каком-то смысле может рассматриваться как деформированный фуллерен $\mathrm{C}_{60}$, электродинамический запрет снимается в силу понижения симметрии и изменения его группы с $Y_{h}$ до $D_{5 h}$. Это проявляется в том, что в спектре фуллерена $\mathrm{C}_{70}$ появляются линии, запрещенные в обычном КР и разрешенные в инфракрасном поглощении.

Как нами было показано, усиление спектра КР в явлении ГКР связано с так называемыми сильным дипольным и квадрупольным взаимодействиями, возникающими в поверхностных электромагнитных полях вблизи ше- роховатой поверхности металла. Усиление дипольного взаимодействия происходит из-за усиления компоненты

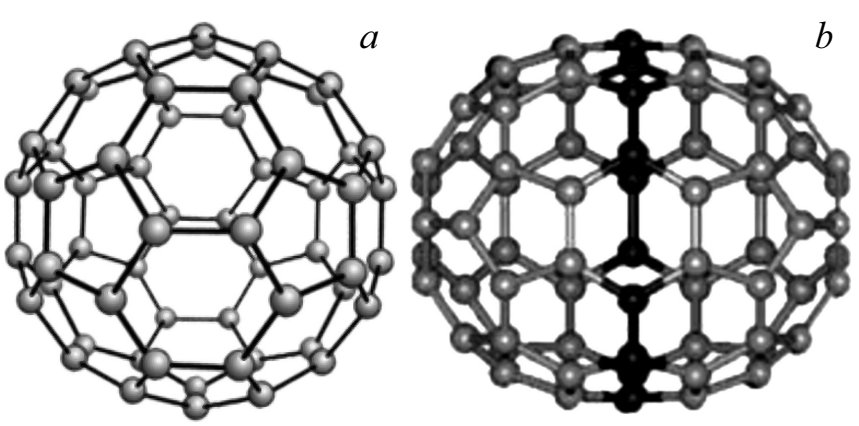

Рис. 1. (a) Фуллерен $\mathrm{C}_{60},(b)$ фуллерен $\mathrm{C}_{70}$.

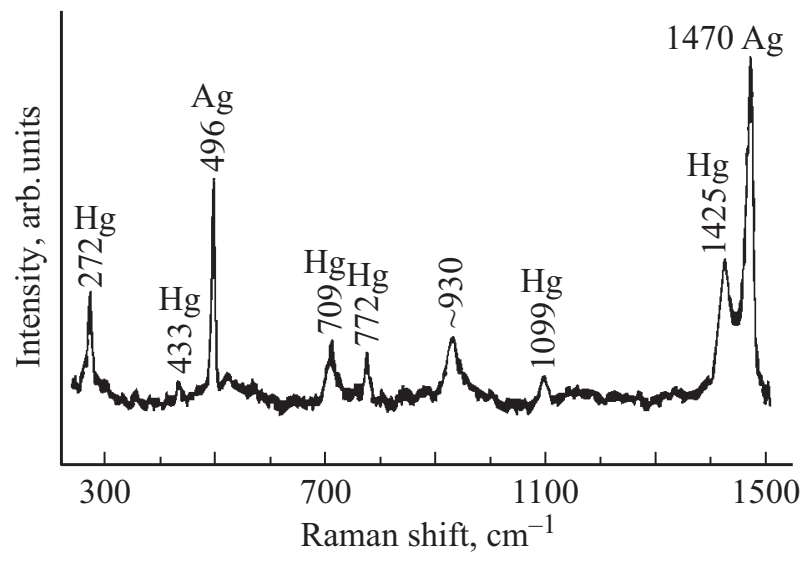

Рис. 2. Спектр ГКР фуллерена $\mathrm{C}_{60}$. Видно, что в спектре наблюдаются только квадрупольно разрешенные линии, обусловленные колебаниями с неприводимыми представлениями $A_{g}$ и $H_{g}$. Дипольно разрешенные линии с неприводимым представлением $T_{1 u}$ отсутствуют. 
напряженности поля $E_{z}$, перпендикулярной поверхности, в то время как возникновение сильного квадрупольного взаимодействия происходит из-за сильного увеличения одноименных производных электрического поля $\frac{\partial E_{i}}{\partial x_{i}}$, а также в связи с особенностями матричных элементов квадрупольных моментов $Q_{e, i i}$, имеющих постоянный знак. Последняя особенность является чисто квантовомеханическим эффектом. Моменты $Q_{e, i i}$ отвечают за сильное квадрупольное взаимодействие, возникающее в системе, и называются основными квадрупольными моментами $Q_{\text {main. }}$ Гамильтониан взаимодействия света с молекулой может быть записан в форме

$$
\hat{H}_{e-r}=|\mathbf{E}| \frac{\left(\mathbf{e}^{*} \mathbf{f}_{e}^{*}\right) e^{i \omega t}+\left(\mathbf{e f}_{e}\right) e^{-i \omega t}}{2},
$$

где

$$
f_{e, i}=d_{e, i}+\frac{1}{2 E_{i}} \sum_{k} \frac{\partial E_{i}}{\partial x_{k}} Q_{e, i k}
$$

есть компонента обобщенного вектора взаимодействия света с молекулой,

$$
\begin{gathered}
d_{e, i}=\sum_{\alpha} e x_{\alpha, i}, \\
Q_{e, i, k}=\sum_{\alpha} e x_{\alpha, i} x_{\alpha, k}
\end{gathered}
$$

- компоненты дипольного и квадрупольного моментов электронов молекулы. Здесь $(i, k)=(x, y, z)$.

В симметричных молекулах удобно перейти от квадрупольных моментов $Q_{e, i i}$ к линейным комбинациям моментов $Q_{e, 1}, Q_{e, 2}$ и $Q_{e, 3}$, преобразующимся по неприводимым представлениям группы симметрии, которые в общем случае имеют вид

$$
\begin{aligned}
& Q_{e, 1}=b_{11} Q_{e, x x}+b_{12} Q_{e, y y}+b_{13} Q_{e, z z}, \\
& Q_{e, 2}=b_{21} Q_{e, x x}+b_{22} Q_{e, y y}+b_{23} Q_{e, z z}, \\
& Q_{e, 3}=b_{31} Q_{e, x x}+b_{32} Q_{e, y y}+b_{33} Q_{e, z z} .
\end{aligned}
$$

При этом моменты $Q_{e, x x}, Q_{e, y y}$ и $Q_{e, z z}$ выражаются через $Q_{e, 1}, Q_{e, 2}$ и $Q_{e, 3}$ в общем случае следующим образом:

$$
\begin{aligned}
& Q_{e, x x}=a_{11} Q_{e, 1}+a_{12} Q_{e, 2}+a_{13} Q_{e, 3}, \\
& Q_{e, y y}=a_{21} Q_{e, 1}+a_{22} Q_{e, 2}+a_{23} Q_{e, 3}, \\
& Q_{e, z z}=a_{31} Q_{e, 1}+a_{32} Q_{e, 2}+a_{33} Q_{e, 3} .
\end{aligned}
$$

Среди моментов $Q_{e, 1}, Q_{e, 2}$ и $Q_{e, 3}$ будут линейные комбинации, существенные для рассеяния, имеющие постоянный знак и преобразующиеся по единичному неприводимому представлению, которые назовем основными квадрупольными моментами $Q_{\text {main }}$, и знакопеременные комбинации, не существенные для рассеяния, преобразующиеся по другим неприводимым представлениям, которые будут называться не основными квадрупольными моментами $Q_{\text {minor }}$ К Как показано в наших публикациях, моменты $Q_{\text {main }}$ в случае молекул достаточно высокой симметрии отвечают за появление запрещенных линий [3]. В симметричных молекулах с группами симметрии $T, T_{d}, T_{h}, O$ и $O_{h}$, а также в фуллерене $\mathrm{C}_{60}$, принадлежащем к икосаэдрической группе $Y_{h}$, линейные комбинации $Q_{e, 1}, Q_{e, 2}$ и $Q_{e, 3}$ имеют вид

$$
\begin{gathered}
Q_{e, 1}=\frac{1}{3}\left(Q_{e, x x}+Q_{e, y y}+Q_{e, z z}\right), \\
Q_{e, 2}=\frac{1}{2}\left(Q_{e, x x}-Q_{e, y y}\right), \\
Q_{e, 3}=\frac{1}{4}\left(Q_{e, x x}+Q_{e, y y}-2 Q_{e, z z}\right) .
\end{gathered}
$$

Здесь основным моментом является $Q_{e, 1}$, преобразующийся по единичному неприводимому представлению, в то время как моменты $Q_{e, 2}$ и $Q_{e, 3}$ являются не основными. Соответственно

$$
\begin{gathered}
Q_{e, x x}=Q_{e, 1}+\frac{2}{3} Q_{e, 3}+Q_{e, 2} \\
Q_{e, y y}=Q_{e, 1}+\frac{2}{3} Q_{e, 3}-Q_{e, 2} \\
Q_{e, z z}=Q_{e, 1}+\frac{2}{3} Q_{e, 3}-2 Q_{e, 2} .
\end{gathered}
$$

Тогда величина $|\mathbf{E}|\left(\mathbf{e f}_{e}\right)$ в выражении (1) для фуллерена $\mathrm{C}_{60}$ может быть представлена как

$$
\begin{aligned}
|\mathbf{E}|\left(\mathbf{e f}_{e}\right) & =\left(\mathbf{E d}_{e}\right)+\frac{1}{2} \operatorname{div} \mathbf{E}\left(Q_{e, 1}+\frac{2}{3} Q_{e, 3}\right) \\
+ & \frac{1}{2}\left(\frac{\partial E_{x}}{\partial x}-\frac{\partial E_{y}}{\partial y}-2 \frac{\partial E_{z}}{\partial z}\right) Q_{e, 2}+\frac{1}{2} \sum_{\substack{i, k \\
i \neq k}} \frac{\partial E_{i}}{\partial x_{k}} Q_{e, i k} .
\end{aligned}
$$

Отсюда видно, что член, содержащий основной момент $Q_{e, 1}$, тождественно обращается в нуль в силу закона электродинамики $\operatorname{div} \mathbf{E}=0$. Это и есть так называемый электродинамический запрет сильного квадрупольного взаимодействия, который возникает благодаря особенностям сильного квадрупольного взаимодействия и принадлежности молекулы к указанным группам симметрии. Поскольку все остальные члены квадрупольного взаимодействия содержат не основные моменты, то указанные оптические процессы определяются только дипольным взаимодействием, и их спектры не будут содержать запрещенные линии. Для фуллерена $\mathrm{C}_{70}$ выражения для моментов $Q_{e, 1}^{\prime}, Q_{e, 2}^{\prime}$ и $Q_{e, 3}^{\prime}$ будут иметь вид

$$
\begin{gathered}
Q_{e, 1}^{\prime}=\frac{1}{2}\left(Q_{e, x x}+Q_{e, y y}\right), \\
Q_{e, 2}^{\prime}=\frac{1}{2}\left(Q_{e, x x}-Q_{e, y y}\right), \\
Q_{e, 3}^{\prime}=Q_{e, z z} .
\end{gathered}
$$

Здесь мы применили штрихованные обозначения, чтобы различать моменты для разных типов фуллеренов. Отметим, что неприводимые представления, характеры и 
соответствующие комбинации дипольных и квадрупольных моментов для группы симметрии $D_{5 h}$, описывающей симметрийные свойства фуллерена $\mathrm{C}_{70}$, представлены в приложении.

Соответствующая величина

$$
\begin{aligned}
& |\mathbf{E}|\left(\mathbf{e f}_{e}\right)=\left(\mathbf{E d}_{e}\right)+\frac{1}{2}\left(\frac{\partial E_{x}}{\partial x}-\frac{\partial E_{y}}{\partial y}\right) Q_{e, 2}^{\prime} \\
& +\frac{1}{2} \frac{\partial E_{z}}{\partial z} Q_{e, 3}^{\prime}-\frac{1}{2} \frac{\partial E_{z}}{\partial z} Q_{e, 1}^{\prime}+\frac{1}{2} \sum_{\substack{i, k \\
i \neq k}} \frac{\partial E_{i}}{\partial x_{k}} Q_{e, i k} .
\end{aligned}
$$

В правой части выражения (3) моменты $Q_{e, 2}^{\prime}$ и $Q_{e, i k}^{\prime}$ являются не основными и не должны влиять на усиление. Однако третий и четвертый члены содержат основные моменты, которые в общем случае должны обусловливать усиление за счет сильного квадрупольного взаимодействия. Можно показать, что эти члены будут несущественны в случае перехода к икосаэдрической группе симметрии $Y_{h}$. Это следует из того, что в этом случае моменты $Q_{e, x x}, Q_{e, y y}$ и $Q_{e, z z}$ преобразуются друг через друга, и в каком-то смысле эквивалентны. Более того, величина $\left(Q_{e, 1}^{\prime}-Q_{e, 3}^{\prime}\right)$ с точностью до множителя $\frac{1}{2}$ совпадает с не основным моментом $Q_{e, 3}$, определенным для группы $Y_{h}(2)$.

В соответствии с дипольно-квадрупольной теорией [3] сечение ГКР для какой-либо колебательной моды определяется суммой вкладов в рассеяние, выражающихся через различные дипольные и квадрупольные моменты, которые мы в дальнейшем будем называть просто $f_{1}$ и $f_{2}$ (рис. 3 ),

$$
\begin{aligned}
& d \sigma_{s, \text { surf }}=\frac{\omega_{\text {inc }} \omega_{\text {scat }}^{3}}{16 \hbar^{2} \varepsilon_{0}^{2} \pi^{2} c^{4}} \frac{\left|\mathbf{E}_{\text {inc }}\right|_{\text {surf }}^{2}}{\left|\mathbf{E}_{\text {inc }}\right|_{\text {vol }}^{2}} \frac{\left|\mathbf{E}_{\text {scat }}\right|_{\text {surf }}^{2}}{\left|\mathbf{E}_{\text {scat }}\right|_{\text {vol }}^{2}} \\
& \times \sum_{p}\left(\begin{array}{c}
\left(V_{(s, p)}+1\right) / 2 \\
V_{(s, p)} / 2
\end{array}\right)\left|T_{d-d}+T_{d-Q}+T_{Q-d}+T_{Q-Q}\right|_{\text {surf }}^{2} d O .
\end{aligned}
$$

Здесь $E_{\text {inc }}$ и $E_{\text {scat }}-$ напряженности падающего и рассеянного электрических полей, значки surf и vol означают, что поле берется на поверхности и в объеме соответственно, $\omega_{\text {inc }}$ и $\omega_{\text {scat }}-$ соответственно частоты этих полей, $V_{(s, p)}$ - колебательное квантовое число вырожденной колебательной моды $(s, p)$, $s$ нумерует группы вырожденных колебаний, $p$ нумерует состояния внутри группы, $T$ обозначает сумму вкладов соответственно диполь-дипольного, дипольквадрупольного, квадруполь-дипольного и квадрупольквадрупольного рассеяний, $\mathrm{dO}$ - элемент телесного угла. Остальные обозначения общепринятые. Здесь мы не выписываем явное выражение для вкладов. Читатель может найти их в монографии [3]. Каждый отдельный вклад подчиняется правилам отбора

$$
\Gamma_{(s, p)} \in \Gamma_{f_{1}} \times \Gamma_{f_{2}} .
$$

Здесь значок Г обозначает неприводимое представление, по которому преобразуются колебательная мода $(s, p)$ и соответственно дипольные и квадрупольные момен-

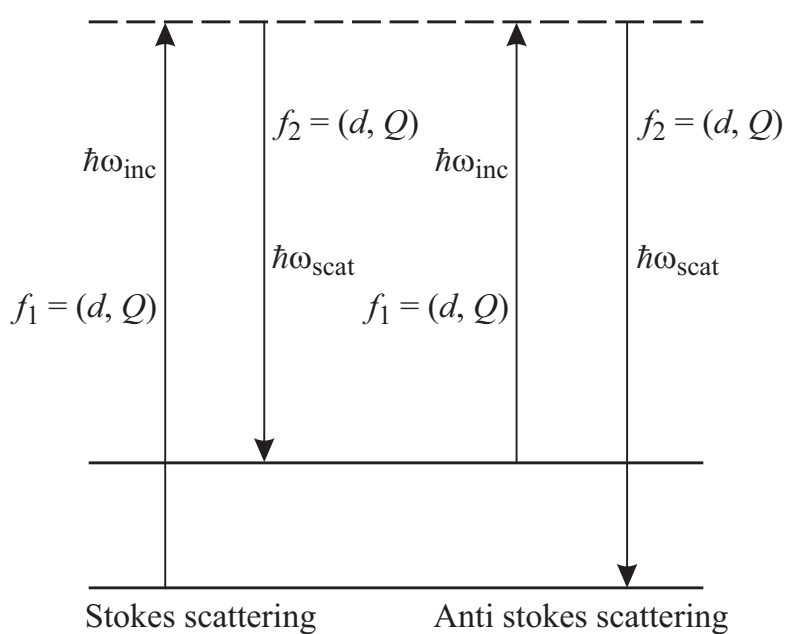

Рис. 3. Диаграмма рассеяния ГКР. Рассеяние происходит через различные комбинации дипольных и квадрупольных моментов $d$ и $Q$.

ты $f_{1}$ и $f_{2}$. В дальнейшем будем обозначать каждый вклад через дипольные и квадрупольные моменты как $\left(f_{1}-f_{2}\right)$. Относительная величина этих вкладов зависит от условий экспериментов. В частности, в случае очень сильной степени шероховатости подложки квадрупольное взаимодействие может быть значительно сильнее, чем дипольное [3] и наибольшее усиление испытывают вклады $\left(Q_{\text {main }}-Q_{\text {main }}\right)$. Согласно правилам отбора $(4)$, эти вклады будут определять линии, обусловленные колебаниями, преобразующимися по единичному неприводимому представлению. Вклады типа $\left(Q_{\text {main }}-d_{i}\right)$ и $\left(d_{i}-Q_{\text {main }}\right)$ также испытывают сильное усиление, но в меньшей степени, чем указанные предыдущие. Эти вклады обусловлены колебаниями, преобразующимися как дипольные моменты $d_{i}$, и определяют появление линий, запрещенных в обычном КР в молекулах с достаточно высокой симметрией. Вклады типа $\left(d_{i}-d_{i}\right)$ и $\left(d_{i}-d_{k}\right) i \neq k$, также могут быть сильно усилены, но в меньшей степени, чем предыдущие два типа. Причем вклад типа $\left(d_{i}-d_{i}\right)$ может при определенных условиях определять интенсивность линий, обусловленных колебаниями, преобразующимися по единичному неприводимому представлению. Однако, как уже указывалось выше, в общем случае относительная величина вкладов зависит от условий эксперимента. Конкретно исследование спектра ГКР фуллерена $\mathrm{C}_{70}$ проводилось в [4]. В табл. 1 указаны волновые числа линий в спектре ГКР $\mathrm{C}_{70}$ и соответствующие им неприводимые представления. При этом, как следует из результатов, наибольшее усиление испытывали линии, обусловленные колебаниями с неприводимыми представлениями $E_{2}^{\prime}$ и $E_{1}^{\prime \prime}$ (рис. 4), которые описывают трансформационные свойства моментов типа $x y, x z$ и $y z$. При этом линии, обусловленные колебаниями с единичным неприводимым представлением $A_{1}^{\prime}$, усилены значительно слабее. Данный факт указывает на то, что дипольное взаимодействие в данном эксперименте значительно сильнее 
Таблица 1. Соотнесение линий спектра ГКР фуллерена $\mathrm{C}_{70}$ неприводимым представлениям группы симметрии $D_{5 h}$, IR active - линии, активные в инфракрасном поглощении

\begin{tabular}{c|c|c|c}
\hline $\begin{array}{c}\text { Волновое } \\
\text { число, } \\
\mathrm{cm}^{-1}\end{array}$ & $\begin{array}{c}\text { Неприводимые } \\
\text { представления } \\
\text { группы } D_{5 h}\end{array}$ & $\begin{array}{c}\text { Волновое } \\
\text { число, } \\
\mathrm{cm}^{-1}\end{array}$ & $\begin{array}{c}\text { Неприводимые } \\
\text { представления } \\
\text { группы } D_{5 h}\end{array}$ \\
\hline 221 & $A_{1}^{\prime}$ & 943 & $E_{1}^{\prime \prime}$ \\
254 & $E_{2}^{\prime}$ & 990 & $E_{2}^{\prime}$ \\
357 & $A_{2}^{\prime \prime}$ IR active & 1031 & $E_{1}^{\prime}$ IR active \\
392 & $E_{2}^{\prime}$ & 1056 & $E_{2}^{\prime}$ \\
410 & $E_{2}^{\prime}$ & 1180 & $E_{1}^{\prime \prime}, E_{2}^{\prime}$ \\
426 & $E_{1}^{\prime \prime}$ & 1212 & $E_{1}^{\prime \prime}, E_{2}^{\prime}$ \\
450 & $A_{1}^{\prime}$ & 1224 & $E_{1}^{\prime \prime}$ \\
500 & $E_{2}^{\prime}$ & 1250 & $E_{1}^{\prime \prime}$ \\
533 & $A_{1}^{\prime}$ & 1281 & $A_{1}^{\prime}$ \\
564 & $E_{1}^{\prime \prime}$ & 1301 & $E_{2}^{\prime}$ \\
574 & $E_{1}^{\prime \prime}$ & 1321 & $A_{1}^{\prime}$ \\
610 & $E_{2}^{\prime}$ & 1337 & $E_{1}^{\prime \prime}$ \\
654 & $E_{1}^{\prime \prime}$ & 1374 & $E_{2}^{\prime}$ \\
697 & $E_{2}^{\prime}$ & 1408 & $E_{1}^{\prime \prime}$ \\
718 & $E_{1}^{\prime \prime}$ & 1441 & $E_{2}^{\prime}$ \\
734 & $A_{1}^{\prime}$ & 1466 & $A_{1}^{\prime}$ \\
787 & $E_{2}^{\prime}$ & 1509 & $E_{1}^{\prime \prime}$ \\
825 & $A_{1}^{\prime}$ & 1563 & $E_{2}^{\prime}$ \\
861 & $E_{2}^{\prime}$ & 1583 & $A_{1}^{\prime}, E_{1}^{\prime \prime}$ \\
893 & $E_{1}^{\prime \prime}$ & & \\
& & &
\end{tabular}

квадрупольного. Значительное усиление линий с неприводимыми представлениями $E_{2}^{\prime}$ и $E_{1}^{\prime \prime}$ обусловлено тем, что в системе координат, связанной с молекулой $\mathrm{C}_{70}$, все три компоненты электрического поля могут быть усилены в связи с тем, что фуллерен $\mathrm{C}_{70}$, по-видимому, может адсорбироваться на металлическую подложку, имея произвольную ориентацию по отношению к поверхности и соответственно к усиленной компоненте поля $E_{z}$, перпендикулярной поверхности. При этом $E_{z}$ может проектироваться на все три оси системы координат, связанной с молекулой, и иметь отличные от нуля компоненты напряженности электрического поля $E_{x}^{\prime}, E_{y}^{\prime}$ и $E_{z}^{\prime}$. Здесь мы ввели обозначения компонент поля со штрихом, тем самым отличая их от компонент поля в системе координат, связанной с поверхностью. Кроме того, в спектре наблюдаются две линии с малой интенсивностью, запрещенные в обычном КР с неприводимыми представлениями $A_{2}^{\prime \prime}$ и $E_{1}^{\prime}$, и волновыми числами 357 и $1031 \mathrm{~cm}^{-1}$. Появление этих линий говорит о появлении квадрупольного взаимодействия в системе и соответственно о снятии электродинамического запрета. Однако их сравнительно малая интенсивность говорит, что квадрупольное взаимодействие достаточно слабо. Это может быть связано с тем, что мы имеем сравнительно слабое нарушение симметрии по отношению к икосаэдрической группе $Y_{h}$. Кроме того, в соответствии с условиями эксперимента в [4] металлическая подложка являлась подложкой зеркального типа и шероховатостями с характерным размером порядка $100 \mathrm{~nm}$. По-

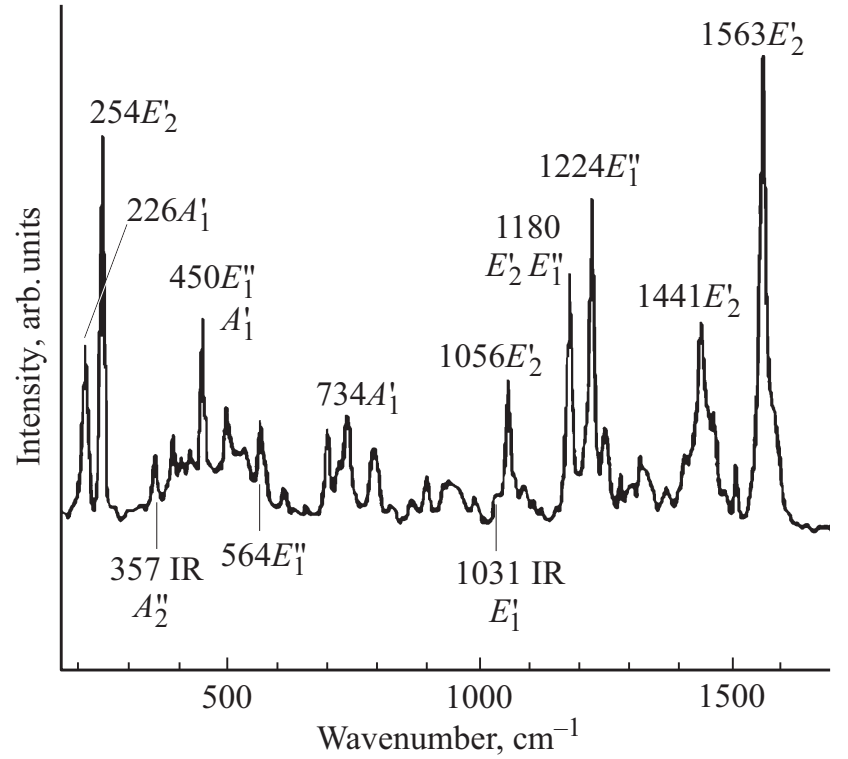

Рис. 4. Спектр ГКР фуллерена $\mathrm{C}_{70}$. Наибольшее усиление имеют линии, обусловленные колебаниями с неприводимыми представлениями $E_{2}^{\prime}$ и $E_{1}^{\prime \prime}$, описывающими трансформационные свойства моментов $x y, x z$ и $y z$.

видимому, при этих условиях шероховатость являлась достаточно „слабой“, что не приводило к большому усилению поля и его производных. Соответственно квадрупольное взаимодействие было меньше, чем дипольное.

Таким образом, в связи с тем, что фуллерен $\mathrm{C}_{70}$ принадлежит к группе $D_{5 h}$ и может в каком-то смысле рассматриваться как деформированный фуллерен $\mathrm{C}_{60}$, растянутый по оси $z$, электродинамический запрет сильного квадрупольного взаимодействия в нем снимается, и в спектре ГКР появляются запрещенные линии. Безусловно, фуллерен $\mathrm{C}_{70}$ отличается от фуллерена $\mathrm{C}_{60}$ количеством атомов, поскольку он может рассматриваться как $\mathrm{C}_{60}$, в который по экватору встроен некоторый ободок из колец атомов углерода. При этом естественно и волновые числа колебаний будут сдвигаться относительно волновых чисел $\mathrm{C}_{60}$. Однако в целом наше качественное рассмотрение имеет определенный смысл. При этом квадрупольное взаимодействие в данном эксперименте, по-видимому, оказывается достаточно слабым, и основную роль здесь играет сильное дипольное взаимодействие, что приводит к наибольшему усилению линий с неприводимыми представлениями $E_{2}^{\prime}$ и $E_{1}^{\prime \prime}$, описывающими трансформационные свойства моментов $x y, x z$ и $y z$, и соответственно обусловленных чисто дипольным рассеянием. Сравнительно малое квадрупольное взаимодействие, по-видимому, также связано с конкретными условиями эксперимента, когда степень шероховатости подложки не очень велика, а также с тем, что „относительная деформация“ $\mathrm{C}_{70}$ по отношению к $\mathrm{C}_{60}$ сравнительно мала. На сравнительно слабое квадрупольное взаимодействие указывает и тот факт, что запрещенные линии в данном эксперименте на $\mathrm{C}_{70}$ имеют сравнительно малую интенсивность. 


\section{Приложение}

Таблица 2. Неприводимые представления группы симметрии $D_{5 h}$, в последнем столбце указаны комбинации дипольных и квадрупольных моментов, преобразующиеся по соответствующим неприводимым представлениям

\begin{tabular}{|c|c|c|c|c|c|c|c|c|c|}
\hline $\begin{array}{l}\text { Неприводимое } \\
\text { представление }\end{array}$ & $C_{1}$ & $2 C_{5}$ & $2 C_{5}^{2}$ & $5 C_{2}$ & $\sigma_{h}$ & $2 S_{5}$ & $2 S_{5}^{3}$ & $5 \sigma_{v}$ & $\begin{array}{c}\text { Комбинация дипольных } \\
\text { и квадрупольных моментов }\end{array}$ \\
\hline$A_{1}^{\prime}$ & 1 & 1 & 1 & 1 & 1 & 1 & 1 & 1 & $\left(Q_{e, x x}+Q_{e, y y}\right), Q_{e, z z}$ \\
\hline$A_{1}^{\prime \prime}$ & 1 & 1 & 1 & 1 & -1 & -1 & -1 & -1 & \\
\hline$A_{2}^{\prime}$ & 1 & 1 & 1 & -1 & 1 & 1 & 1 & -1 & \\
\hline$A_{2}^{\prime \prime}$ & 1 & 1 & 1 & -1 & -1 & -1 & -1 & 1 & $d_{e, z}$ \\
\hline$E_{1}^{\prime}$ & 2 & $2 \cos 72^{\circ}$ & $2 \cos 144^{\circ}$ & 0 & 2 & $2 \cos 72^{\circ}$ & $2 \cos 144^{\circ}$ & 0 & $\left(d_{e, x}, d_{e, y}\right)$ \\
\hline$E_{1}^{\prime \prime}$ & 2 & $2 \cos 72^{\circ}$ & $2 \cos 144^{\circ}$ & 0 & -2 & $-2 \cos 72^{\circ}$ & $-2 \cos 144^{\circ}$ & 0 & $\left(Q_{e, x z}, Q_{e, y z}\right)$ \\
\hline$E_{2}^{\prime}$ & 2 & $2 \cos 144^{\circ}$ & $2 \cos 72^{\circ}$ & 0 & 2 & $2 \cos 144^{\circ}$ & $2 \cos 72^{\circ}$ & 0 & $\left(Q_{e, x x}-Q_{e, y y}, Q_{e, x y}\right)$ \\
\hline$E_{2}^{\prime \prime}$ & 2 & $2 \cos 144^{\circ}$ & $2 \cos 72^{\circ}$ & 0 & -2 & $-2 \cos 144^{\circ}$ & $-2 \cos 72^{\circ}$ & 0 & \\
\hline
\end{tabular}

Примечание. Основные квадрупольные моменты в этой группе будут $Q_{e, 1}^{\prime}=Q_{e, x x}+Q_{e, y y}, Q_{e, 3}^{\prime}=Q_{e, z z}$.

\section{Список литературы}

[1] Полуботко А.М., Челибанов В.П. // ЖЭТФ. 2017. Т. 151. B. 2. C. 305; Polubotko A.M., Chelibanov V.P. // J. Exp. Theor. Phys. 2017. V. 124. N 2. P. 261.

[2] Chelibanov V.P., Polubotko A.M. // Materials Today: Proceedings. 2017. V. 4. P. 6786.

[3] Polubotko A.M. The Dipole-Quadrupole Theory of Surface Enhanced Raman Scattering. NY:: Nova Science Publishers. Inc., 2009. $136 \mathrm{p}$.

[4] Huang Ya-bin, Mo Yu-jun, Mattei G., Pagnanne M., Xie Sishen // Chin. Phys. Lett. 1997. V. 14. N 7. P. 513. 